\title{
Baryon Asymmetry from a Composite Higgs Boson
}

\author{
Sebastian Bruggisser, ${ }^{1}$ Benedict von Harling, ${ }^{1}$ Oleksii Matsedonskyi ${ }^{1}$ and Géraldine Servant ${ }^{1,2}$ \\ ${ }^{1}$ DESY, Notkestraße 85, D-22607 Hamburg, Germany \\ ${ }^{2}$ II. Institute of Theoretical Physics, University of Hamburg, D-22761 Hamburg, Germany
}

(Received 7 May 2018; revised manuscript received 3 August 2018; published 25 September 2018)

\begin{abstract}
We study the nature of the electroweak phase transition (EWPT) in models where the Higgs boson emerges as a pseudo-Nambu-Goldstone boson of an approximate global symmetry of a new strongly interacting sector confining around the $\mathrm{TeV}$ scale. Our analysis focuses for the first time on the case where the EWPT is accompanied by the confinement phase transition of the strong sector. We describe the confinement in terms of the dilaton, the pseudo-Nambu-Goldstone boson of spontaneously broken conformal invariance of the strong sector. The dilaton can either be a mesonlike or a glueball-like state and we demonstrate a significant qualitative difference in their dynamics. We show that the EWPT can naturally be strongly first order, due to the nearly conformal nature of the dilaton potential. Furthermore, we examine the sizable scale variation of the Higgs potential parameters during the EWPT. In particular, we consider in detail the case of a varying top quark Yukawa coupling, and show that the resulting $C P$ violation is sufficient for successful electroweak baryogenesis. We demonstrate that this source of $C P$ violation is compatible with existing flavor and $C P$ constraints. Our scenario can be tested in complementary ways: by measuring the $C P$-odd top Yukawa coupling in electron electric dipole moment experiments, by searching for dilaton production and deviations in Higgs couplings at colliders, and through gravitational waves at LISA.
\end{abstract}

DOI: 10.1103/PhysRevLett.121.131801

Introduction.-Deciphering the origin of the Higgs potential and its stabilization against quantum corrections is an essential step towards the microscopic understanding of electroweak (EW) symmetry breaking. One of very few known options for a natural underlying dynamics is that the Higgs boson is a composite object, a bound state of a new strongly interacting sector that confines around the $\mathrm{TeV}$ scale [1]. The mass gap between the Higgs boson and the yet unobserved other composite resonances can be explained if the Higgs boson is a pseudo-Nambu-Goldstone boson of a global symmetry $G$ of the strong sector that breaks down to a subgroup $H$ due to a strong condensate $\chi$. The Higgs mass is then protected by a shift symmetry.

Another question left unanswered by the standard model (SM) is the origin of the matter-antimatter asymmetry of the Universe. One fascinating framework, the EW baryogenesis mechanism [2,3], fails in the SM due to the absence of a first-order EW phase transition (EWPT) and of sufficient $C P$ violation. Determining the nature of the EWPT is an indispensable step to investigate whether EW baryogenesis is the correct explanation for the baryon asymmetry of the Universe.

Published by the American Physical Society under the terms of the Creative Commons Attribution 4.0 International license. Further distribution of this work must maintain attribution to the author(s) and the published article's title, journal citation, and DOI. Funded by SCOAP ${ }^{3}$.
In composite Higgs $(\mathrm{CH})$ models, since the Higgs boson arises only when a nonzero condensate $\chi$ forms, the confinement phase transition and the EWPT are closely linked. Nevertheless, so far, studies of the EWPT in $\mathrm{CH}$ models considered them separately. They either focused on the confinement phase transition, relying on a fivedimensional description [4-12], or assumed that the EWPT takes place after confinement of the strong sector [13-16]. The novelty of our work is to consider the interlinked dynamics between the Higgs boson and the condensate during the EWPT. We present a detailed analysis of the EWPT associated with the confinement phase transition, within a purely four-dimensional framework, and show that often both phase transitions happen simultaneously. We obtain a strong first-order EWPT, thus solving the first problem of EW baryogenesis in the SM. Complementing previous studies based on five-dimensional-dual models in which the condensate is a glueball, we also treat the meson case (motivated by lattice studies $[17,18]$ ).

An additional attractive feature of $\mathrm{CH}$ models is the explanation of the hierarchy of SM Yukawa couplings as originating from the mixing between elementary and composite fermions $[19,20]$. The resulting Yukawa couplings effectively depend on the confinement scale and are therefore expected to vary during the phase transition. $\mathrm{CH}$ models thus automatically incorporate the possibility of varying Yukawa couplings during the EWPT, which was shown to bring sufficient $C P$ violation for EW baryogenesis [21,22]. Furthermore, the Higgs potential in $\mathrm{CH}$ 
models is intimately tied to the top quark Yukawa coupling. Its variation then leads to a large variation of the Higgs potential, making the coupled Higgs- $\chi$ dynamics nontrivial. We show that sufficient $C P$ violation is naturally induced from the varying top Yukawa coupling, thus solving the second problem of EW baryogenesis in the SM. We therefore demonstrate that $\mathrm{CH}$ models can naturally give rise to EW baryogenesis, even minimal composite Higgs models [23].

Higgs + dilaton phase transition.-The Higgs potential at present times can be parametrized as a sum of trigonometric functions of $h$ [24],

$$
V^{0}[h]=\alpha^{0} \sin ^{2}\left(\frac{h}{f}\right)+\beta^{0} \sin ^{4}\left(\frac{h}{f}\right),
$$

where $\alpha^{0}$ and $\beta^{0}$ are generated by sources that explicitly break $G$ and are fixed to reproduce the mass and vacuum expectation value (VEV) of the Higgs boson. The scale $f$, balancing the Higgs field in the trigonometric functions, is generated by the strong sector condensate. The currently preferred value is around $f=0.8 \mathrm{TeV}$ [25], which we use in the following. The novel aspect of our work is to promote $f$ to a dynamical field. Generally, one expects the confined theory to feature various interconnected condensates, which in particular break the symmetry $G$ (analogous to the chiral symmetry in QCD) with strength given by $f$. Not all of this complex dynamics is necessarily relevant. Flavor physics motivates the strong sector to be nearly conformal above the TeV scale [26]. Confinement is then associated with the spontaneous breaking of conformal invariance. This gives rise to a pseudo-Nambu-Goldstone boson, the dilaton, which we denote as $\chi$. As motivated in Refs. [27-32], once the explicit breaking of conformal invariance is sufficiently small, the dilaton can be significantly lighter than the confinement scale. Its lightness and the fact that its VEV sets all scales in the strong sector then allows us to integrate out other dynamical fields (whose values now become a function of $\chi$ ) and to describe the confinement phase transition in terms of $\chi$ getting a VEV. In particular, this links $f$ to $\chi$. We derive the joint potential for the Higgs boson and the dilaton. The potential (1) is minimized at $h_{0}^{2} \simeq-(1 / 2)\left(\alpha^{0} / \beta^{0}\right) f^{2}$. This suggests that the cosmological evolution of the Higgs boson and the dilaton are tied to each other, and we show under which conditions both fields obtain a VEV simultaneously.

We describe the coupled dynamics of the Higgs boson and the dilaton by using a large- $N$ expansion for the underlying strongly coupled gauge theory [33], where $N$ represents the number of colors. Each insertion of $\chi$ or $h$ is accompanied by a coupling $g_{\chi}$ or $g_{*}$, respectively. By large- $N$ counting, these couplings scale as $\sim 1 / \sqrt{N}$ for mesons and $\sim 1 / N$ for glueballs of the gauge theory. The Higgs boson is expected to be a meson in analogy with QCD pions while for the dilaton both meson and glueball cases are possible.
Requiring a fully strongly interacting theory in the limit $N \rightarrow 1$, this gives [1]

$$
g_{*}=g_{\chi}^{(\text {meson })}=4 \pi / \sqrt{N}, \quad g_{\chi}^{\text {(glueball) }}=4 \pi / N .
$$

The trigonometric functions in $V^{0}[h]$ can be represented as power series in $h / f$. Using the large- $N$ scaling together with dimensional analysis, one finds that this has to correspond to a power series in $g_{*} h /\left(g_{\chi} \chi_{0}\right)$, where $\chi_{0}$ is the dilaton VEV today. This fixes the relation between $f$ and $\chi_{0}$ as $g_{*} f=g_{\chi} \chi_{0}$.

To account for the variation of the scale balancing $h$ in Eq. (1) when $\chi$ varies, the kinetic terms are fixed by dimensional analysis as

$$
\mathcal{L}_{\text {kin }}=\frac{1}{2}\left(\chi / \chi_{0}\right)^{2}\left(\partial_{\mu} h\right)^{2}+\frac{1}{2}\left(\partial_{\mu} \chi\right)^{2} .
$$

We next turn to the Higgs-independent dilaton potential. In an exactly conformal theory, only a term $\chi^{4}$ can appear, which does not allow for a minimum $\chi_{0} \neq 0$. We therefore break conformal invariance explicitly in the UV by a term $\epsilon \mathcal{O}$ in the Lagrangian, where $\mathcal{O}$ is an operator with scaling dimension $4+\gamma_{\epsilon}$. If $0>\gamma_{\epsilon} \gg-1$, the coefficient $\epsilon$ slowly grows when running from the UV scale down to lower energies until it triggers conformal-symmetry breaking and confinement. This is reflected by an additional term in the dilaton potential (see, e.g., [31])

$$
V_{\chi}[\chi]=c_{\chi} g_{\chi}^{2} \chi^{4}-\epsilon[\chi] \chi^{4},
$$

which allows for a minimum at $\chi_{0} \neq 0$. Here the function $\epsilon[\chi]$ is governed by a renormalization group (RG) equation with $\beta$-function $\beta \simeq \gamma_{\epsilon} \epsilon+c_{\epsilon} \epsilon^{2} / g_{\chi}^{2}$ and $c_{\chi}$ and $c_{\epsilon}$ are order-1 coefficients. We trade $\gamma_{\epsilon}$ for the dilaton mass $m_{\chi}$ and fix the remaining constants as $c_{\epsilon}=0.1$, and $c_{\chi}=0.5$ not far from a naive order-1 estimate.

Temperature corrections provide a potential barrier [which the potential (4) does not feature] necessary for a first-order phase transition. Indeed, by dimensional analysis and large- $N$ counting, the free energy of the deconfined phase is given by [4-6]

$$
\Delta V_{T}[\chi=0] \sim-c N^{2} T^{4} .
$$

We choose $c=\pi^{2} / 8$, a value corresponding to $\mathcal{N}=4$ $S U(N)$ super Yang-Mills theory that is representative of a realistic conformal sector. This is modeled by including the standard one-loop thermal corrections from $45 N^{2} / 4$ strongly coupled degrees of freedom with mass $m=g_{\chi} \chi$ [5]. As the temperature drops, $\chi$ eventually tunnels from 0 to the global minimum at $\chi \simeq \chi_{0}$ corresponding to a confined phase. 


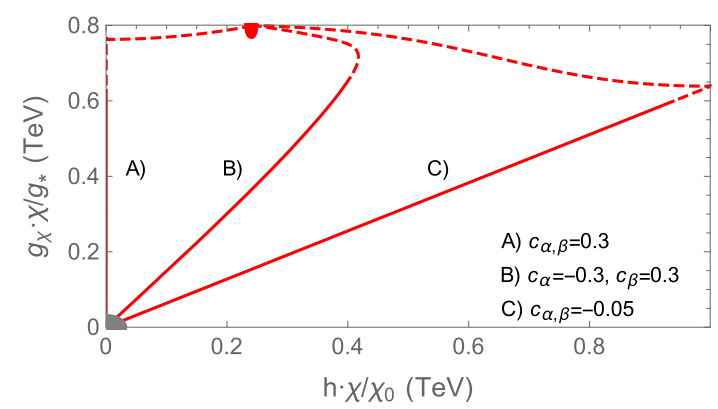

FIG. 1. Transition trajectories for a meson dilaton, $m_{\chi}=700 \mathrm{GeV}, N=3$. Solid lines show the tunneling path to the release point, while dotted lines indicate the subsequent rolling trajectory towards the minimum of the potential at $T_{n}$, indicated by a bullet.

Altogether, the potential of our model reads

$V_{\text {tot }}[h, \chi]=\left(\chi / \chi_{0}\right)^{4} V_{h}^{0}[h]+V_{\chi}[\chi]+\Delta V_{T}^{1-\text { loop }}[h, \chi]$,

where the prefactor $\chi^{4}$ indicates that the dilaton VEV is the only source of mass in the theory. Furthermore, $\Delta V_{T}^{1 \text {-loop }}$ includes the one-loop thermal corrections from SM particles, the Higgs boson and dilaton, as well as the states reproducing the free energy (5).

We have calculated the tunneling trajectory and action for $O(3)$ and $O(4)$-symmetric bubbles in the two-dimensional field space $(h, \chi)$. The phase transition happens at a temperature $T_{n}$ for which the bubble Euclidean action is $S_{E} \approx 140$. In Fig. 1 , we show examples of tunneling trajectories in the meson case. The strength of the phase transition $h\left[T_{n}\right] / T_{n}$, where $h\left[T_{n}\right]$ is at the minimum of the Higgs potential at $T_{n}$, needs to be $\gtrsim 1$, to ensure that sphalerons do not wash out the generated baryon asymmetry. After the phase transition, the system reheats to the temperature $T_{\text {rh }}=\left[30 \Delta V_{\text {tot }} /\left(\pi^{2} g_{\text {dof }}^{\mathrm{SM}}\right)\right]^{1 / 4}$ with $\Delta V_{\text {tot }}$ being the energy difference between the true and false vacuum. We therefore also have to impose that $h\left(T_{\mathrm{rh}}\right) / T_{\mathrm{rh}} \gtrsim 1$. This enforces the light dilaton window. In the left panel of Fig. 2, we show how the phase transition generally quickly becomes supercooled with growing $N$ and decreasing dilaton mass, as found in previous studies of the confinement phase transition focusing on the glueball, e.g., [12]. This effect is much stronger for the glueball than for the meson dilaton due to the different $N$-scaling of its couplings. This disfavors the glueball case as the baryon asymmetry is either washed out or diluted by $\left(T_{\mathrm{rh}} / T_{n}\right)^{3}$ after reheating. We therefore concentrate on the meson case.

We find in the next section that $\alpha^{0}$ and $\beta^{0}$ in Eq. (1) can significantly depend on $\chi$, but this has little impact on the size of the tunneling action. However, it strongly affects the tunneling direction, which controls the size of the $C P$-violating source that we now discuss.

$C P$ violation from varying top mixing.-A sufficient amount of $C P$ asymmetry can be generated during the EWPT from the phase variation of the top quark Yukawa coupling [21]. This $C P$-violating source was considered previously in nonminimal $\mathrm{CH}$ models with additional singlet scalar field [15], and in a five-dimensional model [37]. Here we do not rely on these extra ingredients. In $\mathrm{CH}$ models, the fermion masses originate from linear interactions between the elementary fermions $q_{i}$ and composite sector operators: $y_{i} \bar{q}_{i} \mathcal{O}_{i}$.

The dimensionless coefficients $y_{i}$ are assumed to be of order 1 in the UV, where the mixings are generated. They run subject to an $\mathrm{RG}$ equation with $\beta$-function $\gamma_{i} y_{i}+c_{i} y_{i}^{3} / g_{*}^{2}$, where $c_{i}$ are order- 1 coefficients and the


FIG. 2. Results for the meson dilaton. In the red dashed region, no viable EW minimum can be found or the Higgs-dilaton mixing leads to too large deviations in the Higgs couplings. In the blue dashed region, the baryon asymmetry is washed out after reheating. We also show the cutoff $m_{*}=g_{*} f$. The chosen mass range satisfies current experimental constraints [34]. Left: Phase-transition strength $h\left[T_{n}\right] / T_{n}$. The baryon asymmetry for benchmark point $a(b)$ is $\left|\eta_{B}\right| \times 10^{10} \sim 5-5.5(4-4.5)$. Center: Average Higgs VEV during the phase transition relative to the condensate scale today, $h_{\text {avg }} / f$. Right: Imaginary part of the top Yukawa as a function of the present value of $y / g_{*}^{1 / 2}$ and its anomalous dimension $\gamma_{y}$ for $\left|\beta_{y}\right|=\gamma_{y} y, \arg \beta_{y}=0.1$ and $y_{t L}=\sqrt{g}_{*}$. The current and near future experimental sensitivities correspond respectively to approximately $2 \times 10^{-2}$ [35] and $2 \times 10^{-4}$ [36]. The green bullet indicates the values used for the left and center plots. 
scaling dimension of the operator $\mathcal{O}_{i}$ is given by $5 / 2+\gamma_{i}$. The anomalous dimensions $\gamma_{i}$ can remain sizable over a large energy range due to an approximate conformal symmetry (see, e.g., [26]). The RG evolution stops at the confinement scale $\sim \chi$, where the operators map to composite states. This makes the mixings $y_{i}$ dependent on $\chi$. Integrating out the composite states, one obtains the effective SM Yukawa couplings

$$
\lambda_{q}[\chi] \sim y_{q L}[\chi] y_{q R}[\chi] / g_{*},
$$

where $L$ and $R$ denote the mixings of the left- and righthanded elementary fermions, respectively. In this framework, the SM fermion mass hierarchy is then explained by order-1 differences in the scaling dimensions of the operators $\mathcal{O}_{i}$. This also offers a natural way to make the top Yukawa $\lambda_{t}$ vary during the phase transition, as the condensation scale then changes.

For the $C P$-violating source to be nonvanishing, however, $\lambda_{t}$ needs to vary not only in absolute value but also in phase [21]. To achieve this, we assume that the righthanded top couples to two different operators in the UV,

$$
y_{t R}^{(1)} \bar{t}_{R} \mathcal{O}_{1}+y_{t R}^{(2)} \bar{t}_{R} \mathcal{O}_{2} \Rightarrow \lambda_{t} \sim y_{t L}\left(y_{t R}^{(1)}+y_{t R}^{(2)}\right) / g_{*} \text {. }
$$

Provided that $y_{t R}^{(1,2)}$ are complex and $\mathcal{O}_{1,2}$ have different scaling dimensions (which we assume to be the case), the phase of $\lambda_{t}$ changes with $\chi$. This provides a source of $C P$ violation, but also has another crucial effect on the phase transition that we now explain.

The largest contribution to the Higgs potential in $\mathrm{CH}$ models typically arises from the top quark mixings. We assume that only one of the mixings $y_{t R}^{(1,2)}$, which we denote as $y$, varies sizeably with the dilaton VEV. Its one-loop contribution to the coefficients $\alpha^{0}$ and $\beta^{0}$ in Eq. (1) reads

$\alpha[\chi]=c_{\alpha} \frac{3 y^{2}[\chi] g_{*}^{2}}{(4 \pi)^{2}} f^{4}, \quad \beta[\chi]=c_{\beta} \frac{3 y^{2}[\chi] g_{*}^{2}}{(4 \pi)^{2}} f^{4}\left(\frac{y[\chi]}{g_{*}}\right)^{p_{\beta}}$,

where $c_{\alpha}$ and $c_{\beta}$ are free parameters of our effective field theory, expected to be of order 1. Furthermore, $p_{\beta}=0,2$ depending on the structure of the elementary-composite mixings $[24,38]$ (we choose $p_{\beta}=0$ for definiteness).

Notice that this makes the coefficients explicitly depend on $\chi$. In order to take this into account, we make the replacement [24]

$\alpha^{0} \rightarrow \alpha^{0}+\left(\alpha[\chi]-\alpha\left[\chi_{0}\right]\right), \quad \beta^{0} \rightarrow \beta^{0}+\left(\beta[\chi]-\beta\left[\chi_{0}\right]\right)$

in Eq. (1). Furthermore, since the mixings explicitly break the conformal invariance of the $\mathrm{CH}$ sector, we include an additional contribution $\propto y^{2} \chi^{4}$ in the dilaton potential (which only plays a subdominant role though).
To have the minimum of the Higgs potential at $h_{0} \ll f$ at present times requires that $\left|\alpha^{0} / \beta^{0}\right| \ll 1$. From Eq. (9), on the other hand, we see that generically $|\alpha[\chi] / \beta[\chi]| \gtrsim 1$. This is a manifestation of the well-known tuning required to obtain the observed Higgs mass and VEV in $\mathrm{CH}$ models.

For $\chi$ somewhat away from $\chi_{0}$, the contributions in Eq. (9) typically dominate over $\alpha^{0}$ and $\beta^{0}$ in Eq. (10) and the Higgs potential instead has a global minimum at $h=0$ (for $c_{\alpha, \beta}>0$ ) or $h=f \pi / 2$ (for $c_{\alpha, \beta}<0$ ). This minimum leads to a valley in the Higgs-dilaton potential that can attract the tunneling trajectory during a first-order phase transition. How closely the tunneling trajectory follows this valley is controlled by its relative depth (in particular, determined by $m_{\chi}$ and $N$ ) and the value of $\chi$ for which it becomes deeper than the valley along $h=h_{0}$ that results from the tuned Higgs potential (1) (influenced by $\left.\left|c_{\alpha, \beta}\right|, \gamma_{y}, y[0], y\left[\chi_{0}\right]\right)$. Different tunneling trajectories are shown in Fig. 1. The form of the trajectory has major implications for $\mathrm{EW}$ baryogenesis. In particular, trajectories which closely follow $h=0$ or $h=f \pi / 2$ need to be avoided since the top mass $\propto \sin [h / f]^{1+m} \cos [h / f]^{n}[39]$ (with $m, n$ being model dependent) and thus the $C P$ violating source vanishes along such trajectories (at $h=$ $f \pi / 2$ only if $n \neq 0$ ).

The top mixings are already quite large at $\chi=\chi_{0}$ to ensure a large top Yukawa. Provided that the anomalous dimension $\gamma_{y}$ for the mixing $y$ is negative, it grows for decreasing $\chi$ until it reaches a fixed point whose size is controlled by the constant $c_{y}$ in the $\beta$-function. To obtain a sufficient amount of $y$ variation and $C P$ violation, we choose $\gamma_{y}=-0.3$ and fix $c_{y}$ so that $y[0]=0.4 g_{*}$ in the unbroken phase, while $y\left[\chi_{0}\right]=0.6 \sqrt{\lambda_{t} g_{*}}$ in the broken phase. We also set $c_{\alpha}=-c_{\beta}=-0.3$ in which case the detuned valley is along $h=f \pi / 4$. We have calculated the action for tunneling along straight lines with constant Higgs VEV $h$, which well approximates the exact tunneling paths (cf., Fig. 1). In the central panel of Fig. 2, we plot the

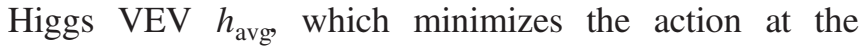
transition temperature. We see that, depending on $m_{\chi}$ and $N$, different trajectories are possible.

Thus, as follows from the first two panels in Fig. 2, the EWPT is strong and our $C P$-violating source is active for a wide range of $m_{\chi}$ and $N$. We have computed the resulting baryon asymmetry using the formalism presented in Ref. [21]. The results are indicated for two benchmark points, assuming a bubble wall velocity of 0.01 (the baryon asymmetry increases by a factor 3-4 if we increase the bubble wall velocity to 0.1 ) and with the varying mixing in Eq. (8) having a complex phase $\arg y_{t R}^{(1)}=\arg y[\chi]=0.1$ and the remaining mixings being fixed as $y_{t R}^{(2)} \simeq 0.4 \sqrt{\lambda_{t} g_{*}}$ and $y_{t L}=\sqrt{\lambda_{t} g_{*}}$.

Note that even for $h\left[T_{n}\right] / T_{n} \gtrsim \mathcal{O}$ (few), we can expect subsonic velocities (needed for baryogenesis) as a sizable friction comes from the large number of degrees of freedom 
becoming massive when they go through the bubble wall. Our baryon asymmetry values (which should only be taken as indicative given order-1 uncertainties) are typically close to the observed value $\eta_{B} \sim 8.5 \times 10^{-11}$. In contrast with phase transitions studied so far, our Higgs VEV grows very large during the EWPT before decreasing, and since $\eta_{B}$ scales as the integral of $(h / T)^{2}$ over the bubble wall, this leads to a large baryon asymmetry. Furthermore, we find that the bubble wall width $L_{w}$ is small, also contributing to a large baryon asymmetry. However, we actually enter a regime where the derivative expansion used in the EW baryogenesis formalism $\left(L_{w} T \gg 1\right)$ [21] starts to break down.

Experimental signatures.-The experimental signatures of our scenario include those related to the transition path and $C P$ violation, and those related to the phase-transition strength. The former are strongly sensitive to the $y$ running. The running mixing $y$ can have a measurable effect on both the Higgs boson and the dilaton phenomenology, as well as on observables that are indirectly sensitive to the couplings of $h$ and $\chi$. Many of these effects arise from the term responsible for the top mass, which in the meson case with $n=0$ reads

$$
\lambda_{t}[\chi] \chi \sin \frac{h}{f} \bar{t}_{L} t_{R} \supset \bar{t}_{L} t_{R} h\left(\lambda_{t}^{0} \frac{\chi}{f}+\beta_{\lambda_{t}} \frac{\chi-f}{f}\right),
$$

where $\lambda_{t}^{0}$ is the SM top Yukawa coupling, and for one varying mixing we have $\beta_{\lambda_{t}} \sim \beta_{y}$ [see Eq. (7)]. $\chi$ and $h$ in this expression are linear combinations of the mass eigenstates. Importantly, $\beta_{\lambda_{t}}$ is complex, as required by the varying Yukawa phase. The highest sensitivity to the resulting complex couplings comes from measurements of the electron electric dipole moment [40]. These restrict the $C P$-odd coupling of the-mass eigenstate-Higgs boson to the top (coming from the $C P$-odd coupling of the-nonmass eigenstate-dilaton) to be $\lesssim 2 \times 10^{-2}$ at $95 \%$ C.L. [35], with a prospect of gaining about 2 orders of magnitude in sensitivity in the near future [36]. In the right panel of Fig. 2, we show how the $C P$-odd $t$ th coupling depends on $y[\chi]$. Forthcoming experiments are expected to probe most of our parameter space.

The strength of the phase transition in our model becomes linked to the dilaton mass, which is light; hence it can be searched for in collider experiments. Higgs-dilaton mixing also leads to observable deviations in the Higgs couplings. Another related signature is a stochastic background of gravitational waves peaked in the milli-Hertz range that can be searched for at LISA [5,41].

In summary, our results strongly support the viability of EW baryogenesis and motivate further studies in concrete calculable realizations of $\mathrm{CH}$ models. In a forthcoming paper [41], we extend this analysis to other possible sources of $C P$ violation.
[1] G. Panico and A. Wulzer, Lect. Notes Phys. 913, 1 (2016).

[2] D. E. Morrissey and M. J. Ramsey-Musolf, New J. Phys. 14, 125003 (2012).

[3] T. Konstandin, Usp. Fiz. Nauk 183, 785 (2013) [Phys. Usp. 56, 747 (2013)].

[4] P. Creminelli, A. Nicolis, and R. Rattazzi, J. High Energy Phys. 03 (2002) 051.

[5] L. Randall and G. Servant, J. High Energy Phys. 05 (2007) 054.

[6] G. Nardini, M. Quiros, and A. Wulzer, J. High Energy Phys. 09 (2007) 077.

[7] B. Hassanain, J. March-Russell, and M. Schvellinger, J. High Energy Phys. 10 (2007) 089.

[8] T. Konstandin, G. Nardini, and M. Quiros, Phys. Rev. D 82, 083513 (2010).

[9] T. Konstandin and G. Servant, J. Cosmol. Astropart. Phys. 12 (2011) 009.

[10] D. Bunk, J. Hubisz, and B. Jain, Eur. Phys. J. C 78, 78 (2018).

[11] B. M. Dillon, B. K. El-Menoufi, S. J. Huber, and J.P. Manuel, arXiv:1708.02953.

[12] B. von Harling and G. Servant, J. High Energy Phys. 01 (2018) 159.

[13] C. Delaunay, C. Grojean, and J. D. Wells, J. High Energy Phys. 04 (2008) 029.

[14] B. Grinstein and M. Trott, Phys. Rev. D 78, 075022 (2008).

[15] J. R. Espinosa, B. Gripaios, T. Konstandin, and F. Riva, J. Cosmol. Astropart. Phys. 01 (2012) 012.

[16] M. Chala, G. Nardini, and I. Sobolev, Phys. Rev. D 94, 055006 (2016).

[17] Y. Aoki et al. (LatKMI), Phys. Rev. D 89, 111502 (2014).

[18] T. Appelquist et al., Phys. Rev. D 93, 114514 (2016).

[19] D. B. Kaplan, Nucl. Phys. B365, 259 (1991).

[20] R. Contino, T. Kramer, M. Son, and R. Sundrum, J. High Energy Phys. 05 (2007) 074.

[21] S. Bruggisser, T. Konstandin, and G. Servant, J. Cosmol. Astropart. Phys. 11 (2017) 034.

[22] G. Servant, Phil. Trans. R. Soc. A 376, 20170124 (2018).

[23] K. Agashe, R. Contino, and A. Pomarol, Nucl. Phys. B719, 165 (2005).

[24] G. Panico, M. Redi, A. Tesi, and A. Wulzer, J. High Energy Phys. 03 (2013) 051.

[25] C. Grojean, O. Matsedonskyi, and G. Panico, J. High Energy Phys. 10 (2013) 160.

[26] R. Contino, in Physics of the large and the small, TASI 09, Proceedings of the Theoretical Advanced Study Institute in Elementary Particle Physics, Boulder, Colorado, USA, 2009 (World Scientific, Singapore, 2011), pp. 235-306.

[27] R. Contino, A. Pomarol, and R. Rattazzi (unpublished).

[28] F. Coradeschi, P. Lodone, D. Pappadopulo, R. Rattazzi, and L. Vitale, J. High Energy Phys. 11 (2013) 057.

[29] B. Bellazzini, C. Csaki, J. Hubisz, J. Serra, and J. Terning, Eur. Phys. J. C 74, 2790 (2014).

[30] Z. Chacko and R. K. Mishra, Phys. Rev. D 87, 115006 (2013).

[31] E. Megias and O. Pujolas, J. High Energy Phys. 08 (2014) 081.

[32] E. Megias, G. Panico, O. Pujolas, and M. Quiros, Nucl. Part. Phys. Proc. 282-284, 194 (2017). 
[33] E. Witten, Nucl. Phys. B160, 57 (1979).

[34] K. Blum, M. Cliche, C. Csaki, and S. J. Lee, J. High Energy Phys. 03 (2015) 099.

[35] V. Cirigliano, W. Dekens, J. de Vries, and E. Mereghetti, Phys. Rev. D 94, 034031 (2016).

[36] K. Kumar, Z.-T. Lu, and M. J. Ramsey-Musolf, in Fundamental Physics at the Intensity Frontier (2013), pp. 159-214, https://inspirehep.net/record/1272872/files/ arXiv:1312.5416.pdf.
[37] B. von Harling and G. Servant, J. High Energy Phys. 05 (2017) 077.

[38] O. Matsedonskyi, G. Panico, and A. Wulzer, J. High Energy Phys. 01 (2013) 164.

[39] A. Pomarol and F. Riva, J. High Energy Phys. 08 (2012) 135.

[40] J. M. Pendlebury et al., Phys. Rev. D 92, 092003 (2015).

[41] S. Bruggisser, B. von Harling, O. Matsedonskyi, and G. Servant, arXiv:1804.07314. 